\title{
INFLUENCIA DEL FENÓMENO DE OSCILACIÓN CUASI-BIENAL EN LAS AFLUENCIAS DEL SISTEMA SUR DE ABASTECIMIENTO DE BOGOTÁ
}

\section{Influence of the Quasi-Biennial Oscillation phenomenon in the affluences of the Bogotá supply system}

\author{
Angie Daniela Aguirre Cardenas ${ }^{1}$, Sebastian Almanza Velasco ${ }^{1}$, Yulia Ivanova ${ }^{2}$ \\ ${ }^{1}$ Universidad Militar Nueva Granada (Bogotá, Colombia). u1101959@unimilitar.edu.co, \\ u1101960@unimilitar.edu.co \\ ${ }^{2}$ M.Sc. Universidad Militar Nueva Granada (Bogotá, Colombia). yulia.ivanova@unimilitar.edu.co
}

(Recibido septiembre 25 de 2017 y aprobado noviembre 20 de 2017)

\begin{abstract}
Resumen
Colombia es uno de los países cuyo régimen hidrológico se encuentra altamente influenciado por diferentes fenómenos macro climáticos. Se ha demostrado que el fenómeno ENSO en algunas regiones del país afecta en un $30 \%$ la escorrentía superficial, esto repercute directamente en las actividades de los sectores sociales, económicos y en las condiciones ambientales de estas zonas. Existen otros fenómenos cuya incidencia sobre la variabilidad hidrológica no se ha estudiado a profundidad, uno de estos es el evento de la Oscilación Cuasi Bienal (OCB). En este estudio se examinó la influencia de este fenómeno sobre las afluencias del sistema de abastecimiento hídrico sur de Bogotá, conformado por los embalses Chisacá y la Regadera, cuya importancia radica en permitir el abastecimiento de agua potable para los habitantes de localidad de Usme, en la cual residen 250.000 personas aproximadamente. Haciendo uso de series de caudales promedios mensuales de estaciones ubicadas aguas arriba de los embalses estudiados y del índice histórico de OCB a $50 \mathrm{hPa}$, se diseñó una metodología basada en el análisis correlativo entre las afluencias mensuales a los embalses y el índice macro climático que caracteriza el fenómeno $\mathrm{OCB}$, y se demostró que este fenómeno se manifiesta con un retardo de 11 a 12 meses en la zona de estudio y que el régimen hídrico en un 10 a $14 \%$ depende de la aparición de este evento climático. Los resultados obtenidos demuestran que la variabilidad hidrológica en la zona del estudio no solo depende del fenómeno del ENSO sino también de la OCB, aunque un menor grado, y debe ser tenida en cuenta en los estudios de la variabilidad hidrológica y modelos predictivos de los fenómenos macroclimáticos debido a su relevancia sobre las actividades productivas dependientes del agua.
\end{abstract}

Palabras clave: Afluencias; embalses; fenómeno Oscilación Cuasi bienal - OCB; variabilidad recurso hídrico.

\begin{abstract}
Colombia is one of the countries whose hydrological regime is highly influenced by different macro-climatic phenomena. It has been shown that the ENSO phenomenon in some regions of the country affects surface runoff by $30 \%$, which has a direct impact on socio-economic activities. There are other phenomena whose incidence on hydrological variability has not been studied in depth. One of these is the event of the Quasi Biennial Oscillation (QBO). The study analyzed the influence of this phenomenon on the influx of the south Bogota system that supplies drinking water to 250,000 people living in the town of Usme. Using a series of monthly average results of stations located upstream of the reservoirs studied and of the historical OCB index at $50 \mathrm{hPa}$, a methodology based on correlative analysis between the monthly inflows to the reservoirs and the macro-climatic index that characterizes the OCB phenomenon. It was shown that this phenomenon manifests itself with a delay of 11 to 12 months and that the water regime in 10 to $14 \%$ depends on the phenomenon of OCB. This demonstrates that hydrological variability depends not only on the ENSO phenomenon but also on the OCB, but to a lesser degree, and must be taken into account in studies of hydrological variability and its incidence on the development of productive activities dependent of water.
\end{abstract}

Keywords: Affluences; reservoirs; Quasi-Biennial Oscillation phenomenon; variability water resource. 


\section{INTRODUCCIÓN}

Colombia está situada sobre el ecuador geográfico, cuenta con una variabilidad climática influenciada por la cuenca Amazónica y la Cordillera de los Andes. La interacción de ambas formaciones y de otros factores de carácter físico - geográfico, inducen la aparición de climas de carácter local y regional de alta complejidad, los cuales dificultan la predicción climática a distintas escalas temporales [1]. Se ha demostrado mediante diferentes investigaciones que los fenómenos macro climáticos como: El Niño y La Niña-Oscilación del Sur (ENOS), la Oscilación del Sur (OS), la Oscilación del Atlántico Norte (NAO) y la Oscilación Cuasi Bienal (OCB), exhiben una alta influencia en la hidroclimatología colombiana [2]. Sin embargo, gran parte de las investigaciones sobre el tema se han enfocado en las fases extremas del ENOS, dejando atrás el estudio de la influencia de otros fenómenos como la OCB. Por esto se hace necesario estudiar el efecto de otros eventos macro climáticos sobre la hidroclimatología colombiana.

La Oscilación Cuasi Bienal (OCB) domina la variabilidad interanual del viento zonal en la estratosfera tropical. El primero en reconocer el fenómeno de OCB fue Ebdon en 1960. Este fenómeno consiste en regímenes de viento zonal alternativos hacia el este y hacia el oeste [3]. Este viento zonal también coincide con el ciclo anual de calentamiento y enfriamiento global en los vientos. El viento proviene del oriente en verano y del occidente en invierno de latitudes polares a tropicales; es así como se observa en la alta troposfera y la estratosfera un vórtice polar estratosférico más frío y más estable durante su fase occidente y un vórtice polar más caliente y más perturbado durante su fase oriente [4].

En 1979, después de estudiar la OCB se establecieron el conjunto de características presentadas por este fenómeno, estas se presentan a continuación: muestra simetría con respecto al ecuador, con regímenes de viento del oriente y occidente alternados regularmente con un periodo aproximado de 27 a 30 meses, se manifiesta primero cerca de los $30 \mathrm{~km}$ de altura y se propaga hacia abajo con una tasa de $1 \mathrm{~km} / \mathrm{mes}$, por lo cual, la magnitud y dirección del viento en la tropopausa está fuera de fase con la magnitud y dirección del viento en la estratosfera media, su amplitud es de aproximadamente $20 \mathrm{~ms}^{-1}$ en el ecuador y decae a la mitad de ese valor cerca de los $12^{\circ}$ de latitud. Su máxima amplitud se encuentra entre los niveles de 23-30 km y desaparece hacia la tropopausa y hacia la estratosfera alta $(50-60 \mathrm{~km})$. Por otra parte, se destaca como los máximos asociados a vientos del oriente son mayores comparados con los del occidente (de 20 a $30 \mathrm{~ms}^{-1}$, comparado con 10 a 15 $\left.\mathrm{ms}^{-1}\right)$ [3].

La OCB influye en la precipitación independientemente de las fases extremas del ENOS, cuando se presentan velocidades altas hacia el occidente se producen excesos de precipitación en todo el territorio colombiano, no obstante, las altas velocidades hacia el oriente generan anomalías de precipitación irregulares y pequeñas, por otro lado, las bajas velocidades en ambas direcciones generan déficit de precipitación para todas las regiones del país. Cuando un evento ENOS ocurre, la OCB interactúa con él generando durante la fase del Niño aumento de las temperaturas mínimas y máximas y durante la fase de la Niña disminución de las mismas [5-6].

De este modo, la predicción de la incidencia de la OCB sobre la hidroclimatología puede llegar a tener importantes implicaciones, porque se ha demostrado en diferentes partes del mundo como la aparición de este evento tiene influencia en los sectores sociales, económicos y en las condiciones ambientales. Un ejemplo, es el efecto presentado en los cultivos de maíz en Sur África, donde la OCB estratosférica actúa para reforzar el aumento de los rendimientos de maíz durante la fase oeste [7]. Otro caso es, la influencia directa de la dirección de los vientos asociados a la OCB, sobre la formación de huracanes en el Atlántico. Esto debido a la manifestación de la OCB en latitudes cerca de los $10^{\circ} \mathrm{N}$ como una disminución del flujo de viento proveniente de la dirección oriente en su fase occidente y un fortalecimiento del flujo de viento del occidente cuando se encuentra en fase oriente. En contraste, cuando se tiene viento débil del este en la estratosfera baja, las condiciones favorecen la convección y por consiguiente la formación de huracanes [3].

En el año 1995, Pérez Suárez buscó establecer con sus estudios, la presencia de una conexión entre la incursión del mar en las costas y la manifestación de distintos fenómenos macro climáticos vinculados con la variabilidad climática. Establecieron que, si existe una relación entre los ciclones tropicales y la actuación de la OCB y el ENOS, el comportamiento de 
las inundaciones costeras producidas por estos eventos también debe estar dominado por las diferentes fases de estos. El flujo del oriente de OCB (fase negativa) y la fase positiva del ENOS impiden la aparición de ciclones en el Atlántico, por lo cual la generación de inundaciones en las costas al paso de ciclones se reduce, sin embargo, con el flujo del occidente de la OCB (fase positiva) y la fase neutral o positiva del ENOS, en los mares interamericanos y el Atlántico la actividad de los ciclones aumenta, fomentada por las corrientes de circulación atmosférica [8].

Igualmente, se cuenta con trabajos donde se demuestran que las condiciones atmosféricas y oceánicas a gran escala, como el fenómeno de OCB, ejercen una influencia considerable en los patrones de frecuencia baja de la variabilidad climática e hidrológica de América del Norte [4]. También hay estudios desarrollados en San Petersburgo, Rusia; enfocados en la predicción estadística de las precipitaciones de África oriental utilizando información de fases de Oscilación Cuasi Bienal. [9]

A nivel nacional las investigaciones en el tema son escasas, pero se cuenta con el de trabajo de Germán Poveda, quién estudió como se ve influenciada la hidroclimatología colombiana y la zona norte de sur América por el fenómeno ENOS, dentro de su investigación también se estudian otros fenómenos macro climáticos de carácter atmosférico y oceánico que tienen influencia sobre esta zona ,como la NAO, OCB y las ondas de Madden-Julian, y como estos se relacionan con el ENOS generando irregularidades hidrológicas [10].

El fenómeno de OCB ha sido estudiado en Colombia, en zonas como el Amazonas y el Pacifico, se cuenta con un estudio donde se evaluó la influencia de la OCB en los eventos cálidos y fríos en el Pacifico colombiano, y se concluyó que la OCB presenta una ciclicidad que al parecer tiene una influencia relacionada con la intensidad y magnitud de la fase cálidas (El Niño) y su opuesta (La Niña) en el Océano Pacífico tropical [8].

Según todo lo mencionado anteriormente, es demostrada la incidencia de la OCB sobre la hidroclimatología en diferentes partes del mundo y parcialmente en Colombia.
De la misma forma, la alta variabilidad hidrológica es producto de la variabilidad climática, de la cual una de las componentes es el fenómeno de la OCB.

Por consiguiente, se hace necesario el estudio de la incidencia de este evento climático sobre el régimen hidrológico, esta investigación considera como caso de estudio el sistema de abastecimiento Sur de la cuidad de Bogotá, este corresponde a la cuenca del Rio Tunjuelo.

La cuenca río Tunjuelo, está conformado por la laguna de los Tunjos, el embalse de Chisacá, el cual regula los caudales de los ríos Chisacá y Mugroso, y por último, el embalse La Regadera, el cual regula los tributarios y además permite la derivación del agua captada hacia la Planta de Tratamiento El Dorado, conformándose de esta manera el Sistema de Abastecimiento Sur de la EAAB, encargado de la potabilización de una porción del agua cruda captada del sistema del río Tunjuelito así como del abastecimiento de agua potable para la localidad de Usme, en la cual residen 250.000 personas aproximadamente [11].

El embalse Chisacá tiene una capacidad de almacenamiento de 6,7 millones de metros cúbicos, con un área aproximada de $85 \mathrm{~km}^{2}$, esta cuenca hidrográfica está compuesta por las subcuencas de los ríos Chisacá, Mugroso y Curibital. El embalse La Regadera está ubicado a 5,5 $\mathrm{Km}$ de Usme, tiene un volumen de almacenamiento de 3,8 millones de metros cúbicos en un área aproximada de $167 \mathrm{~km}^{2}$. Estos embalses se encuentran en la parte alta del río Tunjuelo, y su importancia radica en su posición geoestratégica privilegiada para un abastecimiento por gravedad a la zona sur de Bogotá [12].

En la Figura 1, se presenta un esquema de la zona de estudio, donde se destacan los afluentes de ambos embalses y la ubicación de las estaciones limnimétricas empleadas para la recolección de datos.

Conociendo la influencia de la OCB en la hidroclimatología, se podrán establecer a futuro, modelos predictivos de los fenómenos macro climáticos estructurados para usar este fenómeno como variable predictora de las condiciones que se presentarán a largo plazo. 


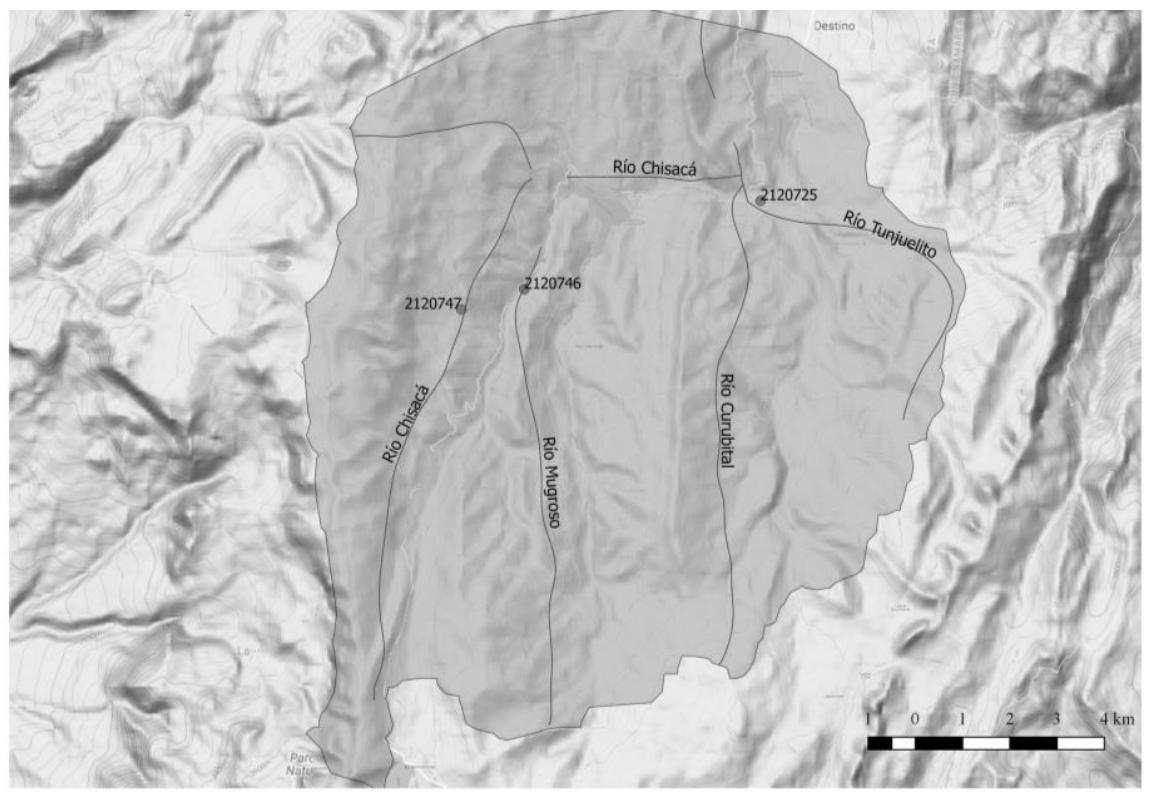

Figura 1. Esquema de la zona de estudio

\section{METOdOLOGÍA}

En la Figura 2, se presenta gráficamente la metodología empleada para identificar la incidencia del fenómeno de la OCB sobre el régimen hídrico en el sistema sur de abastecimiento de Bogotá.
Se recopiló la información de series históricas de caudales promedios mensuales de tres estaciones ubicadas aguas arriba de los embalses estudiados (la ubicación de las estaciones hidrológicas se puede apreciar en la Figura 1). Estas series se obtuvieron de la Corporación Autónoma Regional de Cundinamarca (CAR) y el Instituto de Hidrología, Meteorología y

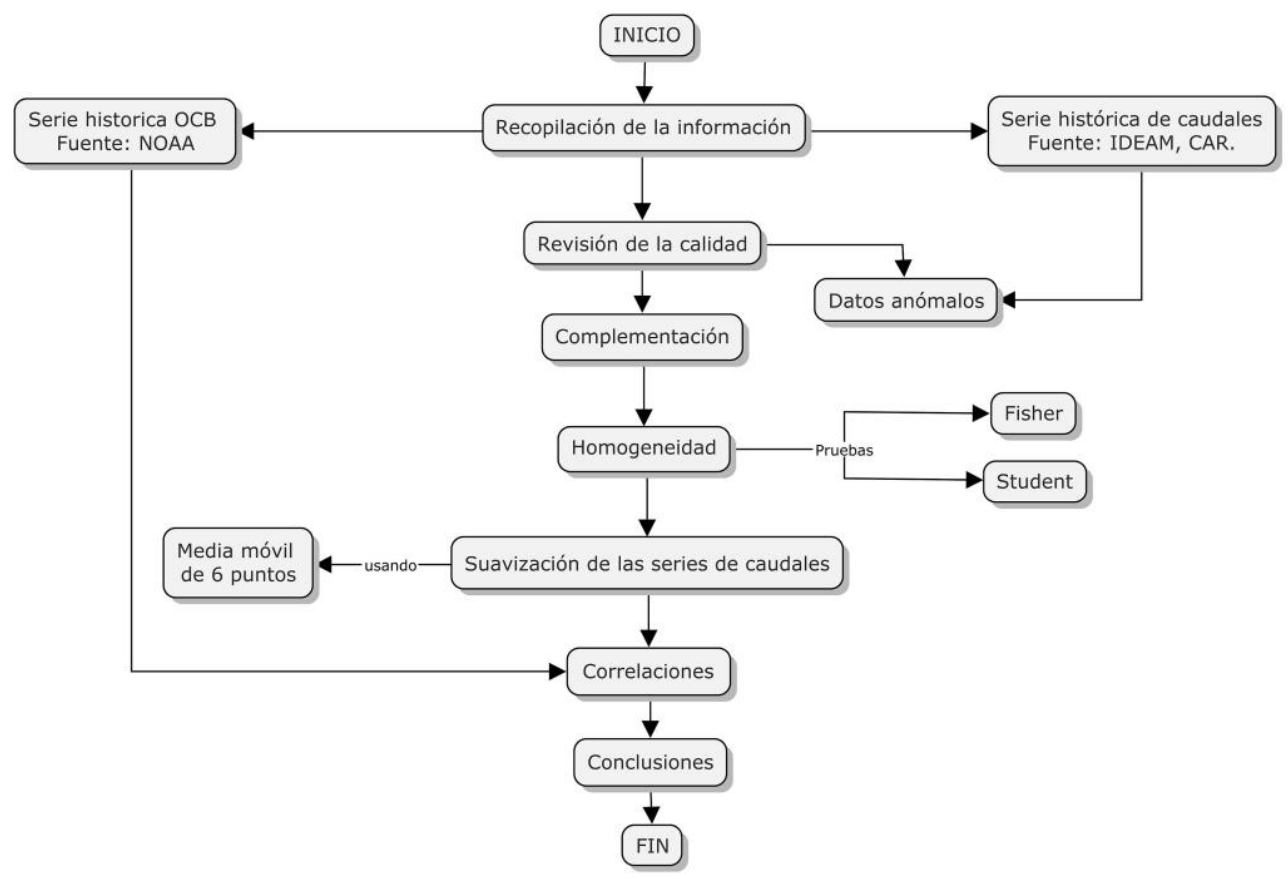

Figura 2. Metodología aplicada en el estudio. 
Estudios Ambientales (IDEAM). Los registros históricos del índice de la Oscilación Cuasi Bienal a 50 hP se obtuvieron de la Administración Nacional Oceánica y Atmosférica (NOAA).

Generalmente los estudios sobre la variabilidad climática deben realizarse usando series de datos con un periodo de registro mayor de 30 años, sin embargo, las estaciones ubicadas en la zona de estudio no contaban con series de datos con el periodo de tiempo requerido, por esta razón, se trabajaron series con un periodo de registro mayor de 20 años (registro común de 1991 a 2013), buscando que estos reflejaran el comportamiento de la variable en el tiempo y que a su vez fuesen representativos para poder determinar un patrón de comportamiento. Se trabajaron registros de 3 estaciones, la información correspondiente a cada una de estas se encuentra en la Tabla 1.

Tabla 1. Información de las estaciones

\begin{tabular}{ccccc}
\hline Código & $\begin{array}{c}\text { Nombre } \\
\text { estación }\end{array}$ & Tipo & $\begin{array}{c}\text { Entidad } \\
\text { operadora }\end{array}$ & $\begin{array}{c}\text { Período } \\
\text { registro }\end{array}$ \\
\hline 2120725 & Rio Curibital & LM & CAR & $1991-2013$ \\
2120747 & Rio Mugroso & LM & CAR & $1991-2013$ \\
2120746 & La Toma & LM & IDEAM & $1991-2013$ \\
\hline
\end{tabular}

Inicialmente de las series históricas de caudales fueron eliminados los datos anómalos utilizando el criterio estadístico de Grubbs [13]. Posteriormente las series de caudales fueron complementadas usando el análisis regresivo empleando las series completas de caudales de las estaciones geográficamente más cercanas y utilizando el método de correlación lineal [14].

Seguido a esto, se revisó la homogeneidad de las series de datos para verificar que cada una conformara un conjunto estadístico válido. La homogeneidad de una serie temporal de una variable se analiza por medio de pruebas, orientadas a garantizar que las alteraciones presentes en las series son el resultado de procesos meteorológicos y climáticos, y no sean ocasionadas por factores externos; la verificación de homogeneidad se realizó usando los criterios de Student y Fischer, donde el primero revisa la homogeneidad por los promedios de las series temporales y el segundo por la varianza de las series temporales.

Se procede a realizar la suavización de la serie de datos utilizando la media móvil, una técnica empleada para suavizar los picos máximos y mínimos de las series para de este modo lograr obtener mejores correlaciones entre el índice de OCB y las series de caudales [13]. Las series históricas de caudales promedios mensuales de cada una de las estaciones, fueron suavizadas mediante media móvil en seis puntos, dado que al usar esta media móvil se encontraron mejores correlaciones estadísticamente significativas en comparación con las obtenidas con la media móvil de 3 y 4 puntos.

Por último, se realizó un análisis de correlación entre la serie temporal ya suavizada de cada estación con la serie histórica de OCB $50 \mathrm{hPa}$. Estas correlaciones fueron construidas con los desfases de 1 hasta 12 meses, considerando que los fenómenos macroclimáticos tardan en manifestarse en función de aspectos como su intensidad y las características de territorio.

Se verificó la correlación y significancia entre las series de caudales suavizadas y el índice de OCB a 50 hPa a partir de una regresión lineal. Se identificó para cada estación el mes donde se encontraba la mayor correlación significativamente estadística y de esta manera con el desfase de tiempo hasta el mes identificado, se logró establecer el desfase de tiempo con el cual se refleja la OCB en los cuerpos hídricos de la zona de estudio.

\section{RESULTADOS}

Los resultados obtenidos en el estudio se muestran a continuación. En total se han presentado 21 datos anómalos en las series de caudales medios mensuales estos fueron eliminados de los registros. Una vez se obtuvieron los registros sin datos anómalos, estos fueron complementados a través del análisis regresivo logrando registros continuos de 22 años correspondientes al periodo de 1991 a 2013. Los registros fueron revisados por homogeneidad aplicando ambas pruebas, donde todos los datos fueron encontrados como homogéneos, esto indica que los valores de las series temporales no presentan cambios bruscos en sus valores, su distribución 
presenta poca variabilidad indicando la existencia de poca dispersión, por lo tanto, los datos son confiables y pueden ser usados para cálculos y predicciones. Los resultados de datos anómalos y homogeneidad por ambas pruebas se presentan en la Tabla 2.

Tabla 2. Datos anómalos y homogeneidad

\begin{tabular}{cccc}
\hline \multirow{2}{*}{$\begin{array}{c}\text { Código } \\
\text { Estación }\end{array}$} & $\begin{array}{c}\text { Datos } \\
\text { Anómalos }\end{array}$ & \begin{tabular}{c} 
Homogeneidad \\
\cline { 3 - 4 } Student
\end{tabular} & $\begin{array}{c}\text { Prueba } \\
\text { Fisher }\end{array}$ \\
\hline 2120747 & 9 & Homogénea & Homogénea \\
2120725 & 7 & Homogénea & Homogénea \\
2120746 & 5 & Homogénea & Homogénea \\
\hline
\end{tabular}

La Tabla 3 muestra los resultados obtenidos de la suavización de las series de caudales promedios mensuales de cada una de las estaciones, usando media móvil de 3, 4 y 6 puntos. En conclusión, con los 3 tipos de media móvil se obtenían correlaciones estadísticamente significativas, pero con el uso de la media móvil de 6 puntos estas correlaciones son en promedio $24 \%$ más altas de las obtenidas con la media móvil de 3 y 4 puntos.

Tabla 3. Comparación entre medias móviles

\begin{tabular}{|c|c|c|c|c|}
\hline \multirow{2}{*}{ Código } & \multirow{2}{*}{ Parámetro } & \multicolumn{3}{|c|}{ Media Móvil } \\
\hline & & MV 3 & MV 4 & MV 6 \\
\hline \multirow{3}{*}{2120747} & $\begin{array}{l}\text { Mes mayor } \\
\text { correlación }\end{array}$ & Enero & Marzo & Febrero \\
\hline & Correlación & 0,08 & 0,09 & 0,10 \\
\hline & $\begin{array}{l}\text { Significancia } \\
\text { estadística }\end{array}$ & SI & SI & SI \\
\hline \multirow{3}{*}{2120725} & $\begin{array}{l}\text { Mes mayor } \\
\text { correlación }\end{array}$ & Noviembre & Diciembre & Noviembre \\
\hline & Correlación & 0,08 & 0,08 & 0,10 \\
\hline & $\begin{array}{c}\text { Significancia } \\
\text { estadística }\end{array}$ & $\mathrm{SI}$ & $\mathrm{SI}$ & $\mathrm{SI}$ \\
\hline \multirow{3}{*}{2120746} & $\begin{array}{l}\text { Mes mayor } \\
\text { correlación }\end{array}$ & Octubre & Noviembre & Diciembre \\
\hline & Correlación & 0,11 & 0,11 & 0,14 \\
\hline & $\begin{array}{c}\text { Significancia } \\
\text { estadística }\end{array}$ & $\mathrm{SI}$ & $\mathrm{SI}$ & SI \\
\hline
\end{tabular}

En la Tabla 5 se presentan los resultados obtenidos de las correlaciones lineales entre el índice OCB 50 reportado por la NOAA y las series de datos suavizadas, además se muestran cuales correlaciones son estadísticamente significativas y cuáles no.
Como resultado se obtuvo que el $64 \%$ de las correlaciones son significativas estadísticamente. Esto permite evidenciar, la incidencia del fenómeno de OCB sobre la variabilidad hidrológica de las afluencias al sistema sur de abastecimiento de la ciudad de Bogotá.

Se realizó un desfase de doce meses respecto a la fecha de registro del índice de OCB 50 de lo cual se pudo observar como en promedio el fenómeno tiene periodo de manifestación sobre el régimen hídrico de 11 a 12 meses, se concluye esto debido que las mayores correlaciones que presentaron en el mes de noviembre y diciembre (Tabla 4). También se estableció si la correlación entre las series de datos y

\begin{tabular}{ccc}
\hline Estación & Mes & $\begin{array}{c}\text { Mayor valor coeficiente de } \\
\text { correlación }\end{array}$ \\
\hline 2120747 & Febrero & 0,101 \\
2120725 & Noviembre & 0,105 \\
2120746 & Diciembre & 0,142 \\
\hline
\end{tabular}

el índice OCB 50 es significativa, es decir, que, aunque el coeficiente de correlación no sea numéricamente alto la relación puede resultar significativa entre las dos series.

Tabla 4. Meses donde se obtuvieron las mayores correlaciones

En las Figuras 3, 4 y 5, se presenta la relación entre las series de caudales suavizadas, el OCB-50 hPa y el tiempo de registro, para cada una de las series de datos aplicando la media móvil de 6 puntos y teniendo en cuenta el mes donde se produjo la mayor correlación.

De las gráficas presentadas, se puede analizar la forma en la cual durante la fase positiva del fenómeno de OCB $50 \mathrm{hPa}$ hay un incremento en los caudales de los afluentes a los embalses, reflejando de este modo un patrón repetitivo en las tres estaciones, en los caudales de los meses donde se obtuvo la mayor correlación.

\section{CONCLUSIONES}

A través de los análisis realizados se puede concluir que el fenómeno de la OCB efectivamente tiene incidencia sobre las afluencias a los embalses de la Regadera y Chisacá. Los resultados de las correlaciones confirman por un lado la significancia de estas y por el otro como el régimen hídrico de los 
afluentes a los embalses analizados depende en un 10 a $14 \%$ de la OCB.

Con respecto al tiempo de manifestación del fenómeno sobre los cuerpos hídricos, se encontró, que el fenómeno de OCB tarda en manifestarse un periodo de 11 a 12 meses sobre los caudales aportantes al sistema de abastecimiento sur de Bogotá. En la mayor parte de los casos, durante la fase positiva del OCB se produce un incremento en el caudal de los afluentes a los embalses estudiados.

Los resultados obtenidos pueden ser usados para predecir las condiciones de caudal presentes en los embalses en el Sistema Sur de Abastecimiento de Bogotá, usando como una de las variables predictivas el fenómeno de OCB.

Tabla 5. Correlaciones OCB 50 con series de datos suavizados
Finalmente, se concluye como la integración del fenómeno OCB a los estudios de la variabilidad hidrológica puede mejorar la eficiencia de los modelos predictivos de las afluencias a los embalses, que hasta el momento solo consideran la influencia del fenómeno ENSO como la única variable de índole macro climática.

\begin{tabular}{|c|c|c|c|c|c|c|c|c|c|c|c|c|}
\hline \multicolumn{13}{|c|}{ Rio Mugroso OCB 50 - 2120747} \\
\hline Mes & ENE & FEB & MAR & $A B R$ & MAY & JUN & JUL & AGO & SEP & OCT & NOV & DIC \\
\hline $\begin{array}{l}\text { Valor coeficiente } \\
\text { correlación }\end{array}$ & 0,010 & 0,101 & 0,090 & 0,087 & 0,089 & 0,092 & 0,091 & 0,082 & 0,066 & 0,041 & 0,009 & 0,026 \\
\hline Significancia & $\mathrm{Si}$ & $\mathrm{Si}$ & Si & $\mathrm{Si}$ & Si & $\mathrm{Si}$ & $\mathrm{Si}$ & $\mathrm{Si}$ & $\mathrm{Si}$ & No & No & No \\
\hline \multicolumn{13}{|c|}{ Rio Curibital OCB 50 - 2120725} \\
\hline Mes & ENE & FEB & MAR & $A B R$ & MAY & JUN & JUL & AGO & SEP & OCT & NOV & DIC \\
\hline $\begin{array}{l}\text { Valor coeficiente } \\
\text { correlación }\end{array}$ & 0,009 & 0,002 & 0,015 & 0,025 & 0,034 & 0,045 & 0,057 & 0,071 & 0,085 & 0,098 & 0,105 & 0,100 \\
\hline Significancia & No & No & No & No & No & Si & $\mathrm{Si}$ & $\mathrm{Si}$ & $\mathrm{Si}$ & $\mathrm{Si}$ & Si & $\mathrm{Si}$ \\
\hline \multicolumn{13}{|c|}{ La Toma OCB 50 - 2120746} \\
\hline Mes & ENE & FEB & MAR & $A B R$ & MAY & JUN & JUL & AGO & SEP & OCT & NOV & DIC \\
\hline $\begin{array}{l}\text { Valor coeficiente } \\
\text { correlación }\end{array}$ & 0,014 & 0,055 & 0,082 & 0,090 & 0,073 & 0,035 & 0,012 & 0,056 & 0,094 & 0,123 & 0,140 & 0,142 \\
\hline Significancia & No & $\mathrm{Si}$ & Si & $\mathrm{Si}$ & Si & No & No & Si & Si & $\mathrm{Si}$ & Si & Si \\
\hline
\end{tabular}

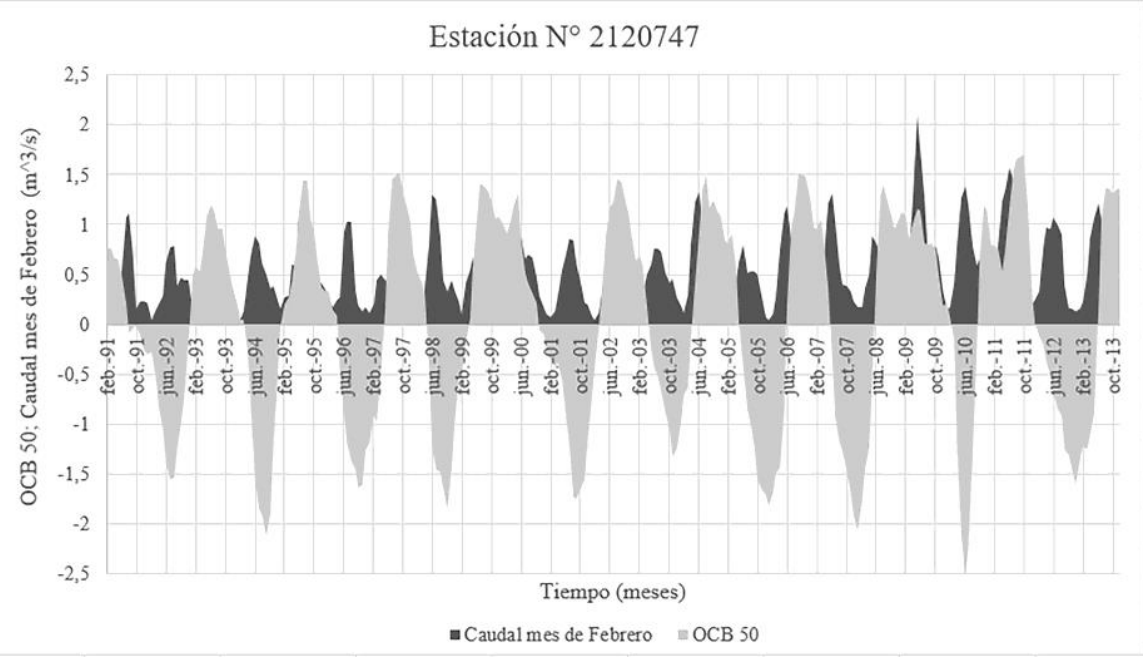

Figura 3. Relación de la serie de OCB 50 con el caudal del mes de febrero, donde se obtuvo la mayor correlación para la 


\section{Estación $\mathrm{N}^{\circ} 2120746$}

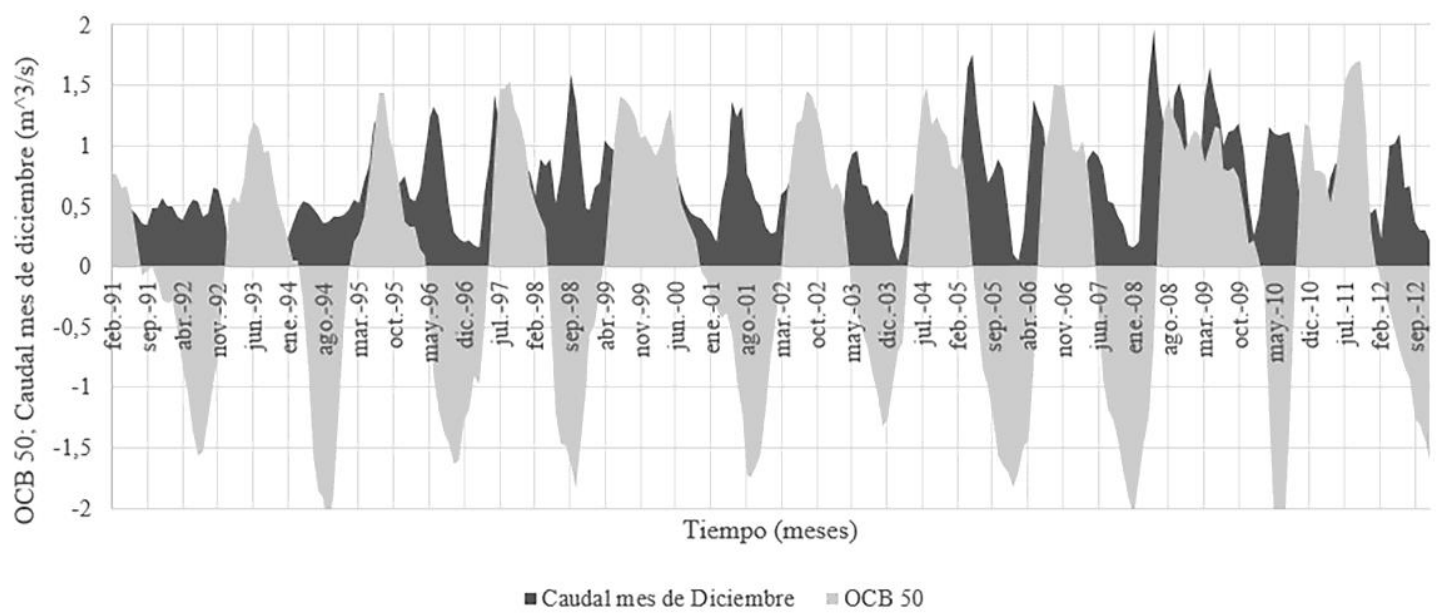

Figura 4. Relación de la serie de OCB 50 con el caudal del mes de noviembre, donde se obtuvo la mayor correlación para la estación

\section{Estación $N^{\circ} 2120725$}

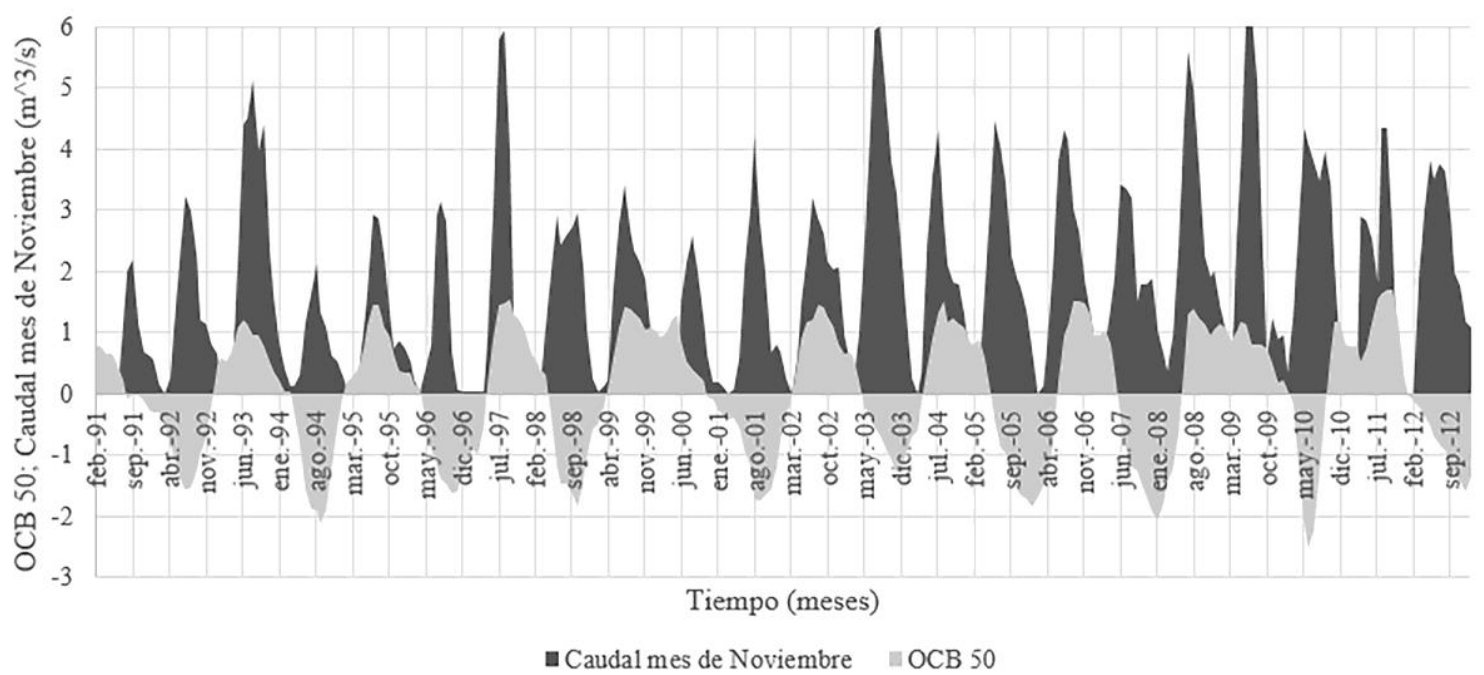

Figura 5. Relación de la seria de OCB 50 con el caudal del mes de diciembre, donde se obtuvo la mayor correlación para la estación

\section{REFERENCIAS}

[1] German, P. (2004). La hidroclimatologia de Colombia: una sintesis desde la escala inter-decadal hasta la escala diurna. Acad. Colom.Cienc., 210-222.
[2] Cortés, M. (2012). Análisis de variables de caudal y precipitación de sectores en los departamentos de Boyacá y Casanare y su alteración por fenómenosmacroclimáticos y de cambio global. L'esprit ing, 3 Vol. 
[3]Amador, J. A. (1996). La Oscilación Cuasi-Bienal, ENOS y acoplamiento de algunos parámetros superficiales y estratosféricos sobre Costa Rica. Topografía y Meteorología Oceanográfica 3 , 45 - 53.

[4] Hansen, F., \& Matthes, K. \&. (2016). Tropospheric QBO-ENSO Interactions and Differences between the Atlantic and Pacific. Journal of Climate, Vol. 29 Issue 4, 1353-1368. DOI: https://doi.org/10.1175/JCLI-D15-0164.1

[5] Martelo, M., Moreno, O., \& Mendoza, C. (2010). Estudio del impacto del cambio climático sobre la agricultura y la seguridad alimentaria en la República Bolivariana de Venezuela. Dirección de Hidrología y Meteorología, Ministerio del Ambiente y los Recursos Naturales.

[6] Cárdenas, P., C. Gil y García, L. (2002). Impacto de los eventos El Niño - Oscilación del Sur en Venezuela. Parte I. Corporación Andina de Fomento, CAF. Caracas, Venezuela.

[7] Mark R, J. (2001). Economic Impacts of Climate Variability in South Africa and Development of Resource Prediction Models . Journal of applied Meteorology, Vol. 41.

[8] Pérez Parrado R., R. J. (1995). Efectos del evento ENOS durante la temporada invernal en Cuba. Boletín SOMETCUBA, Vol. 1, No.2.

[9]Hashim K. Ng'ongolo, S. P. (2010). The statistical prediction of East African rainfalls using quasibiennial oscillation phases information. Scientific Research Publishing, Vol.2.

[10] Jaramillo, G. P. (1998). Retroalimentación dinámica entre el fenómeno el Niño-Oscilacion del Sur y la hidrologia Colombiana. Medellin, Colombia: Universidad Nacional de Colombia.

[11] Orhuela, J. Y. (2016). Evaluación limnológica del embalse la regadera, sus tributarios y la zona de descarga del río tunjuelo. Usme - cundinamarca. Bogota D.C: Universidad Militar Nueva Granada.

[12]Ernesto Torres Quintero, M. C. (2009). Diagnóstico Ambiental de las Cuencas Hidrograficas de Embalses en Colombia. AVANCES Investigación en Ingeniería, Vol.10.
[13] Devore, J. L. (2004). Probabilidad y Estadistica para ingenieria y ciencias. San Luis: Thomson.

[14] Santiago Fernández Fernández, J. M. (2002). Estadística descriptiva. Madrid: ESIC. 